\title{
Thermal Characterization of a Heat Transport System for Satellite Application
}

\author{
Paul Knipper, Sebastian Meinicke, Thomas Wetzel \\ Karlsruhe Institute of Technology \\ Kaiserstraße 12, Karlsruhe, Germany \\ paul.knipper@kit.edu; sebastian.meinicke@kit.edu; thomas.wetzel@kit.edu
}

\begin{abstract}
This contribution presents an approach for a mathematical model which is based on a detailed experimental characterization of a heat transport system as used in common satellite applications. The heat transported is being emitted from the electrical consumers inside the satellite. The present heat transport system consists of two loop heat pipes (LHP), which transport the heat obtained from four arterial heat pipes (AHP) to the heat sink outside the satellite using ammonia as a working fluid. Due to nonconstant operation parameters, e.g. the thermal load and temperature of the heat sink, unwanted oscillations of the saturation temperature can be observed, which have a great impact on the operation temperature of the electrical consumers. In order to eliminate these oscillations and to ensure an optimal operation temperature an additional heat source, e.g. a heating film located on the compensation chamber of the LHPs, is being introduced into the system. This approach requires a fundamental knowledge of the thermal characteristics as well as a reliable control of the heating film. Therefore a mathematical model has been set up based on available concepts in literature and validated using experimental data from a novel test facility. This procedure allows the description of unknown thermal properties by setting up steady state test conditions within the heat transport system. In a second step effects concerning the thermal capacity of the system as well as of the surroundings can be quantified and included into the numerical description of the system. Present results for both, the experimental characterization and the mathematical description, will be presented and compared in detail. Furthermore a brief summary of the knowledge gained from both concepts will be given.
\end{abstract}

Keywords: loop heat pipe, thermal characterization, mathematical model

\section{Introduction}

Loop heat pipes (LHP) are common heat transfer devices used within the aerospace industry. Due to their capability of transferring heat over long distances and their excellent heat transfer performance LHPs are predestined for use in spacecraft thermal control systems as they rely on a self-contained and passive working principle [1]. A schematic view of a typical LHP is given in Figure 1.

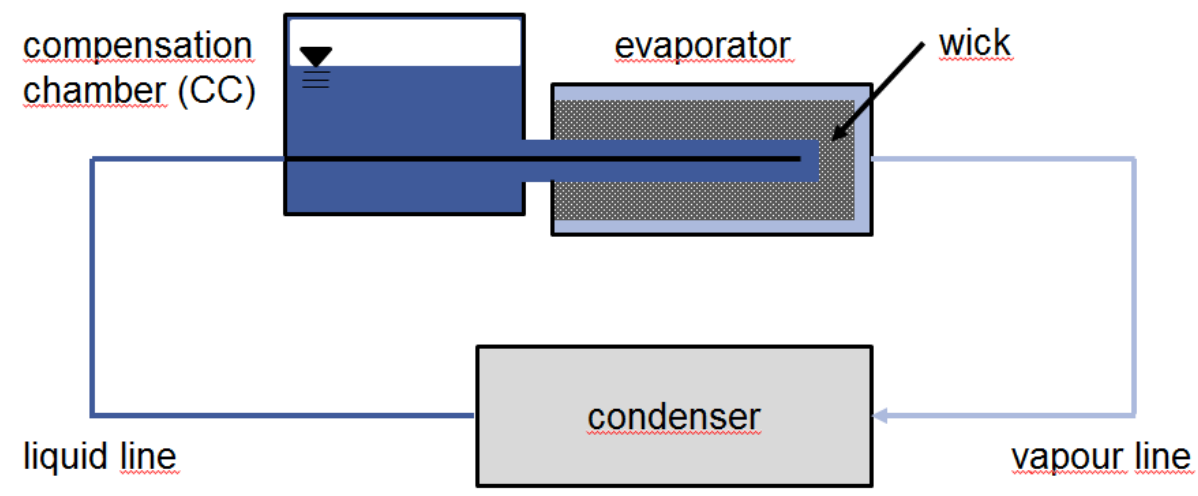

Fig. 1: Schematic representation of a loop heat pipe (LHP). 
Based on a standard refrigeration cycle, the main difference can be found within the evaporator core and the compensation chamber (CC). A so called wick-structure, consisting of a primary and secondary wick, connects the evaporator and the CC and sustains the total pressure drop of the LHP. The task of the secondary wick is to ensure a continuous supply of the primary wick with liquid. In steady-state operation conditions, this is given by the liquid flow returning from the condenser. In transient states the secondary wick is forced to either guide the surplus liquid into the compensation chamber or to retain it. Furthermore, vapour bubbles possibly forming in the evaporator core are being led away from the primary wick in order to prevent a blockage and interruption of the liquid supply. The evaporation occurs within the primary wick and the vapour is led towards the vapour line inside so called vapour grooves, which can be found on the interface between the primary wick and the surrounding pipe wall.

The operation temperature respectively the saturation temperature of the LHP is being influenced by the thermal load as well as the sink temperature [2]. As the sink temperature can be very low for space applications, an additional opportunity to control the saturation temperature is needed. Though several possibilities are described in literature [2], only an approach which does not require a change of the design of the LHP is suitable for this work. Thus, the proposed heating film on the compensation chamber provides an efficient way to control the saturation temperature with little additional effort regarding the electrical power required [3]. However, controlling this heating film is a challenge as its configuration should be based on a reliable mathematical description of the underlying thermodynamics. In regards to the application a lean approach is required to minimize the computing effort and to enable real-time calculations during the application. Therefore, a 1D-model featuring all components of the LHP has been set up. As previous investigations have shown a more detailed approach for the condenser as well as the wick structure including the compensation chamber is essential. The influence of the heat leak connecting the evaporator and the CC is of great interest as it affects the saturation temperature. As only major geometrical parameters are known in detail, the experimental data will be used to extend the mathematical model for the unknown parameters. In order to develop a reliable model, the structure and thermal properties of the primary and secondary wick have to be determined precisely. As no manufacturer specifications are available on their specific properties, a pragmatic approach based on literature and an empirical fitting of experimental data has been adopted and will be presented in the following.

\section{Experimental Setup}

In order to perform a detailed thermal characterization of the present heat transport system, a novel test facility has been set up. The underlying operation parameters used for characterization tests are shown in Table 1.

Table 1. Operation parameters.

\begin{tabular}{|l|l|}
\hline thermal load (AHP) [W] & $9-50$ \\
\hline thermal load (heating film) [W] & $0-10$ \\
\hline sink temperature $\left[{ }^{\circ} \mathrm{C}\right]$ & $-35-20$ \\
\hline
\end{tabular}

According to this wide range of different parameters representative experimental data can be obtained. The test facility consists of two main elements. First there are the two LHPs, which receive the heat to be dissipated from the arterial heat pipes which are linked thermally to their evaporator component. The arterial heat pipes themselves are being heated by using heating tapes. This allows a precise control of the thermal load. The heat sink is being represented by the condenser including a section of sub-cooling for usual operation conditions. As the condenser consists of five cooling panels, which include the pipes of each LHP in a parallel layout, an interaction between both LHPs can't be ruled out completely. This applies as well for the possible interactions between the vapour and liquid lines of each LHP, which are only separated by few millimetres though the highest temperature gradient occurs between both parts. The thermal characterization of the LHP is performed exclusively by using Pt100 surface temperature sensors. All other properties such as mass flow, saturation pressure have to be derived from the temperatures measured. As referring to before, each compensation chamber has been fitted with a heating film for this study, allowing to add an additional heat power of $10 \mathrm{~W}$ per CC.

Second there is the cooling cycle which is connected to the LHPs along the cooling panels featuring countercurrent flow and heat transfer conditions. The cooling cycle consists of a thermostat (Lauda RP855C) to provide the desired sink temperature and a gear pump to control the mass flow of the coolant. Inlet and outlet temperatures of the 
coolant at the condenser are being determined by using Pt100 sheath resistance thermometers. The mass flow is measured with a Coriolis mass flow meter (Siemens FCS400). A schematic overview of the test facility is given in Figure 2 . The LHPs are displayed in red, the arterial heat pipes in green and the cooling cycle in blue.

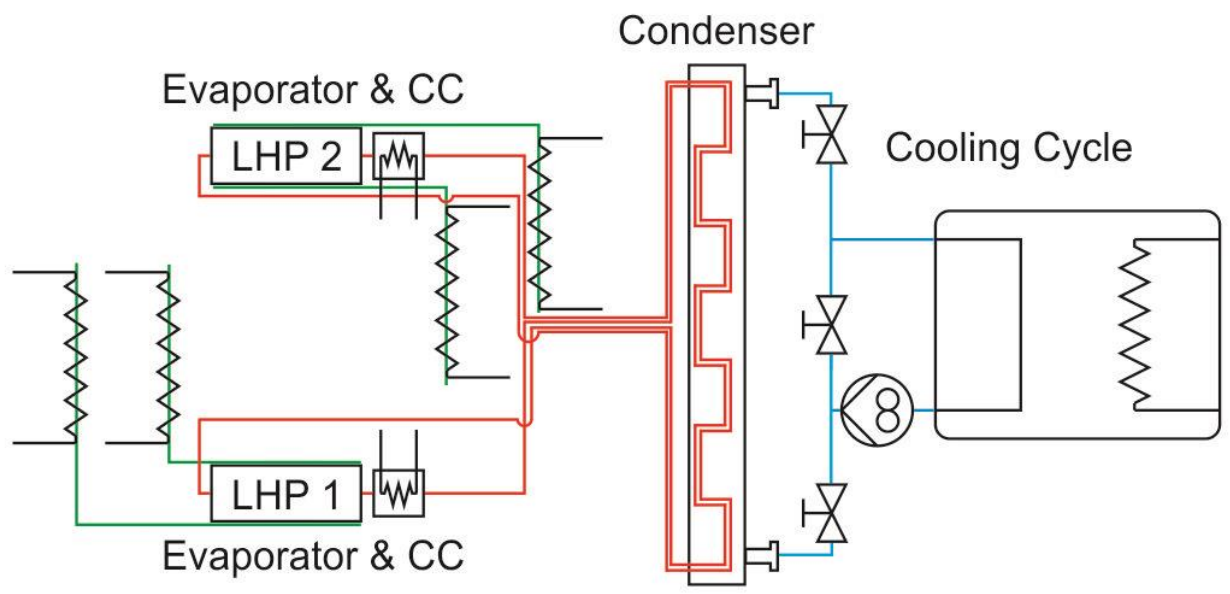

Fig. 2: Schematic representation of the test facility.

In order to evaluate the recorded data in detail, a decent analysis of the uncertainty in measurement has been performed. This procedure is based on the "Guide to the expression of uncertainty in measurement" as described in the ISO/IEC Guide 98-3:2008 [4]. One of the major uncertainties in measurement results from the fact that the fluid temperatures of the LHP are measured by using surface temperature sensors. Therefore, discrepancies to the real value of the fluid temperature have to be taken into account due to the thermal resistances of the considered component. Furthermore, the thermal conductivity of the solid parts of the condenser might have an influence on the temperature determined as high temperature gradients between the ammonia and the coolant are present. Obviously, the temperature sensors themselves only provide a specific accuracy, which is stated at $\pm 0.15 \mathrm{~K}$ by the manufacturer.

\section{Mathematical Model}

The mathematical model of the LHP has to meet several requirements, which result from the proposed application. Thermal energy balances have been formulated separately for all principal components of the LHP (i.e. compensation chamber, evaporator, vapour line, liquid line and condenser), yielding a system of equations to predict the relevant operation temperatures of the LHP. This unified approach considers all internal convective enthalpy fluxes and conductive heat fluxes, the heat losses to the ambient, the effects of the external influencing variables $\dot{Q}_{\text {load }}$ (thermal load) and $T_{\text {Sink }}$ (heat sink temperature) and of the control variable $\dot{Q}_{\mathrm{CC}}$ (electrical heating of compensation chamber). The resulting equation system is arranged in vector form which is indicated by Eq. (1):

$$
\frac{d \vec{T}}{d t}=\overline{\bar{A}}(\dot{m}, \vec{T}) \cdot \vec{T}+\vec{C}\left(\dot{m}, \vec{T}, \dot{Q}_{\text {load }}, T_{\text {Sink }}, \dot{Q}_{\mathrm{CC}}\right)
$$

The general solution of this equation system is a highly non-linear problem which is especially due to the dependency of the components of the matrix $\overline{\bar{A}}$ and of the vector $\vec{C}$ on the ammonia mass flow $\dot{m}$. Furthermore, $\dot{m}$ is not constant throughout the whole internal ammonia cycle of the LHP which is due to the possibility of storing mass in the reservoir and implies a complicated solution procedure for the general, transient application case. This work focusses on the set-up of a steady state model where $d \vec{T} / d t=0$ and $\dot{m}$ is a constant parameter which may be accessed easily by balancing the condenser. If, moreover, temperature dependencies of the fluid properties are neglected, Eq. (1) transforms into a system of linear equations. If $\vec{T}=\left(T_{\mathrm{CC}, \text { in }}, T_{\mathrm{CC}}, T_{\mathrm{ev}}, T_{\text {cond,in }}\right)^{T}$, this system can be easily solved by standard solvers available from the used software MATLAB®. However, a more detailed approach has to be applied for the condenser analysis. This 
component is divided into $N$ discrete, equally sized segments. For each segment, energy conservation equations are again formulated for both the ammonium and the cooling cycle. The specification of appropriate heat transfer kinetics for the counter-current operation of the condenser, as proposed in the experimental setup, allows accessing the desired local temperature courses and phase distributions inside the component. By applying this approach, the complex single-phase and two-phase conditions prevailing inside the condensation pipe are adequately taken into consideration for the calculation of the missing temperature, $T_{\text {cond,out }}$ The determination of the matrix and vector components $(\overline{\bar{A}}, \vec{C})$ requires further knowledge about the effective heat transfer resistances occurring inside the LHP system. We have quantified them in terms of effective heat transfer capabilities $(k A)_{i}$ which are introduced for different components of the LHP $(i=\{$ 'vapor line', 'liquid line', 'wick'\}) and determined from the mean heat transfer kinetic according to Eq. (2):

$$
(k A)_{i}=\frac{\dot{Q}_{\mathrm{i}}}{\Delta T_{\mathrm{LM}, \mathrm{i}}}
$$

where $\dot{Q}_{\mathrm{i}}$ is the transferred heat flow and $\Delta T_{\mathrm{LM}, \mathrm{i}}$ is the mean logarithmic temperature difference whose definition depends on the specific heat transfer case in the component considered. $\dot{Q}_{\mathrm{i}}$ and $\Delta T_{\mathrm{LM}, \mathrm{i}}$ may be determined experimentally by a smart arrangement of the respective temperature sensors. Effective $(k A)_{i}$ values can be fitted for various operating conditions and, thus, reproduce and convey the missing information on the LHP characteristics which is not directly accessible by the manufacturer's specification. The modelling approach presented can be used both to recalculate the relevant temperatures to be compared with corresponding experimental data and to predict control values under certain operating conditions.

\section{Results}

In the following, a brief review of the experimental results will be given. Subsequently, the data will be compared to the results from the mathematical model.

\subsection{Experimental Results}

The operation characteristics recorded for the present LHP are in good accordance with common references in literature as shown in figure 3 [5,6]. For low thermal loads, low mass flow rates or high residence times of ammonia in the liquid line, respectively, result in an increased heat input from the warmer ambient which, in turn, causes the compensation chamber temperature to rise. For medium values of the thermal load, the operation temperature of the LHP reaches its lowest level and rises again for higher thermal loads. Results in figure 3 also indicate that the constant conductance range is not completely reached for the thermal load values investigated in this study which means that the heat transfer capacity of the condenser is oversized in relation to the maximum possible thermal load in the present application case. In addition the compensation chamber temperature is being influenced significantly by the given sink temperature.

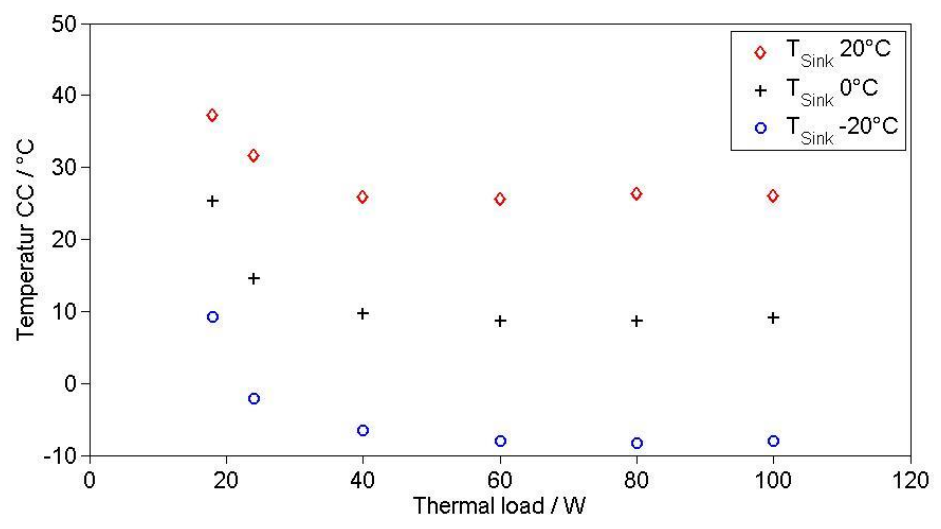

Fig. 3: Comparison of the compensation chamber temperature for different sink temperatures $\left(-20^{\circ} \mathrm{C}, 0^{\circ} \mathrm{C}, 20^{\circ} \mathrm{C}\right)$ and different thermal loads $(19-100 \mathrm{~W})$. 
Furthermore a detailed representation of the main temperatures of the compensation chamber and evaporator shows a consistent distribution for these temperatures. In figure 4 a sink temperature of 0 up to $20{ }^{\circ} \mathrm{C}$ has been chosen as well as a constant thermal load of $80 \mathrm{~W}$ for one LHP.

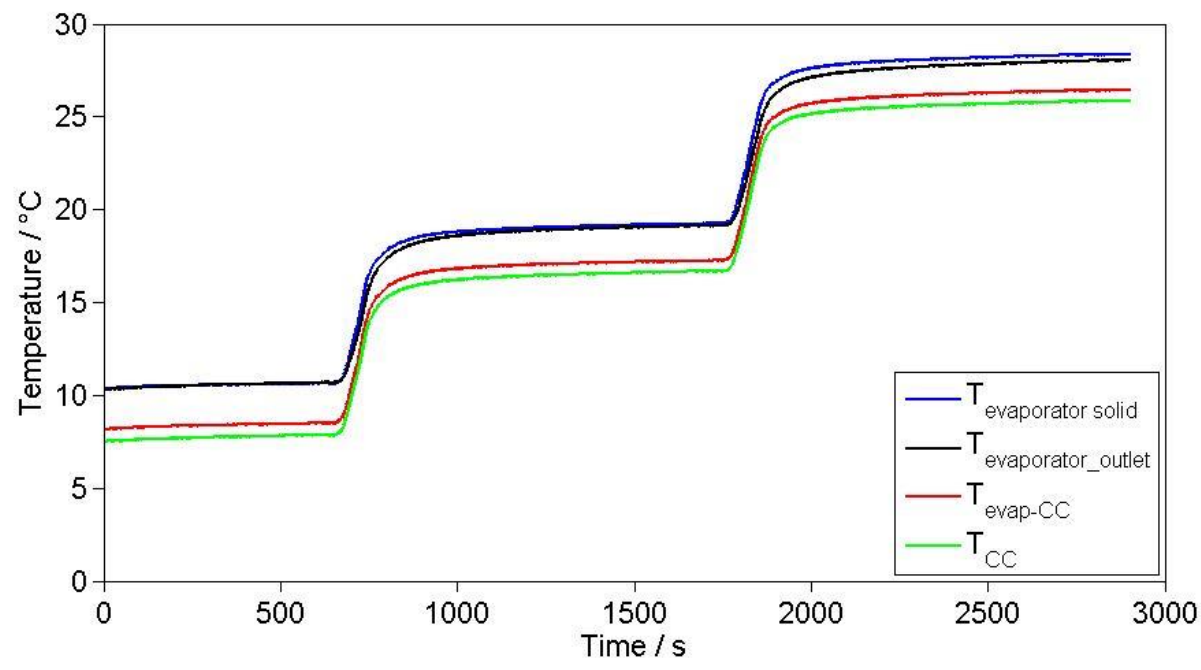

Fig. 4: Comparison of several temperatures along the compensation chamber and evaporator for different sink temperatures $\left(0^{\circ} \mathrm{C}, 10^{\circ} \mathrm{C}\right.$, $\left.20^{\circ} \mathrm{C}\right)$ and a constant thermal load $(80 \mathrm{~W})$.

A constant temperature difference between the compensation chamber and the evaporator has been observed for steady state conditions, as could be expected from similar investigations in the literature (e.g. [7]). For transition periods between two different steady-state operating points the temperature difference is being reduced. But even in the case of an increasing sink temperature of $\Delta \mathrm{T}=10{ }^{\circ} \mathrm{C}$, the present order is being remained, which is required to ensure the stable operation of the LHP. Experimental investigations have shown that a fast and high rise of the sink temperature can lead to a shutdown of the LHP due the heat capacity of the evaporator and surroundings compared to the heat capacity of the compensation chamber. As the intended application of the LHP requires a tight temperature range a shutdown and a following start-up of the LHP should be avoided as this usually results in comparatively high temperature fluctuations. Furthermore, the need of considering the thermal capacity of the future application in the mathematical model is likely in regards to the fact that for now only the stripped LHP has been investigated.

\subsection{Comparison with the Mathematical Model}

In the following, three characteristic temperatures of the LHP - at the condenser outlet, the evaporator outlet, and the compensation chamber - will be studied in detail to compare the mathematical model with the experimental data. Using the modelling approach proposed in section 3 and the effective heat transfer capabilities derived from the experimental data, the mathematical model yields the desired temperature values for fixed combinations of the input parameters $\dot{Q}_{\text {load }}$ and $T_{\text {Sink }}$. The results are shown exemplarily for $T_{\text {Sink }}=0^{\circ} \mathrm{C}$ and $\dot{Q}_{\text {load }}$ values varied between 18 and $100 \mathrm{~W}$ in figure 5 . 


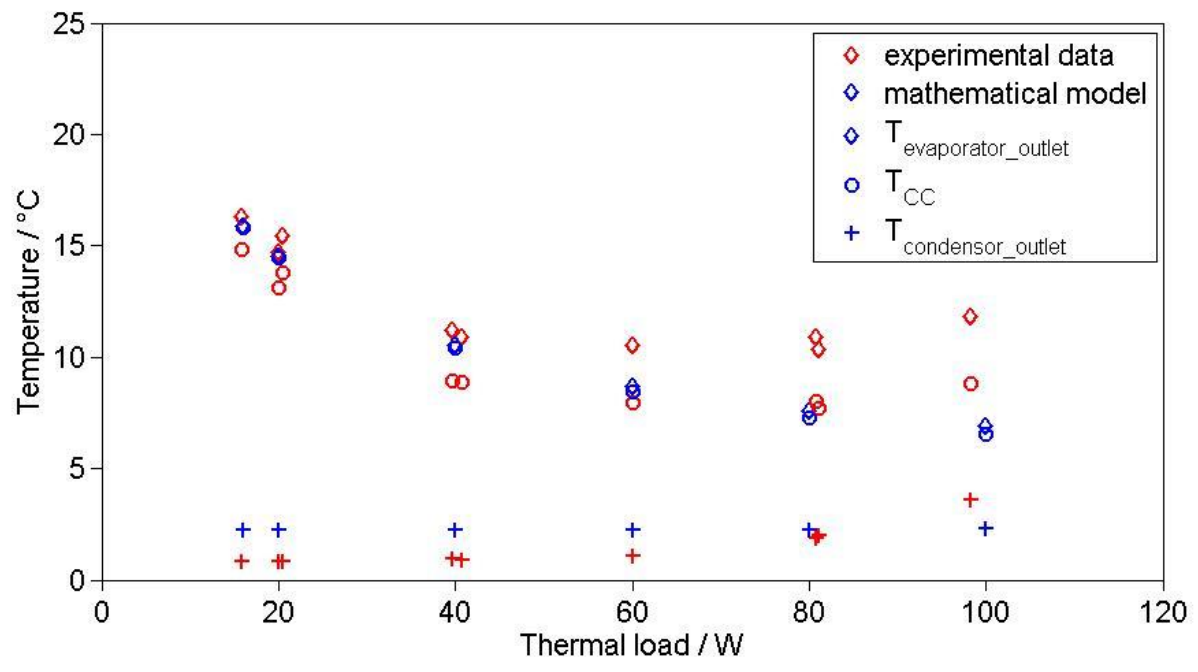

Fig. 5: Comparison of the experimental data and the mathematical mode for the temperatures of the compensation chamber, evaporator and condenser for a sink temperature of $0{ }^{\circ} \mathrm{C}$ and thermal loads of 18 to $100 \mathrm{~W}$.

It can be noted that the both, the experimental and numerical results agree well in their trends for all three temperatures. For high thermal loads, the deviation of the mathematical model to the experimental data increases. Furthermore, a rise of the condenser outlet temperature observed in the experiment cannot be reproduced with the mathematical approach at the moment. Another discrepancy can be observed by considering the temperature difference between the evaporator outlet and the compensation chamber. For the experimental data, one can always note a higher temperature gradient compared to the theoretical data which might be due to the inadequate reproduction of the complex heat transfer processes in the porous wick by the current approach. Nevertheless, for a wide range of the operation conditions a good accordance of the temperature level inside the evaporator and compensation chamber can be stated for both approaches. The tendency of a higher saturation temperature for low thermal loads can be reproduced although the rise of the saturation temperature for a higher thermal load is not being expressed well by the mathematical model yet. This, however, is likely to result from the rather constant value of the condenser outlet temperature. Further optimization and validation of the derived variables of the experiment will be necessary in order to adapt the mathematical model to the actual heat transfer system.

\section{Conclusion}

Within this contribution, a combined experimental and mathematical approach to characterize a heat transport system consisting of two loop heat pipes has been presented. The experimental test loop has been set up carefully to ensure reliability of the temperature data recorded. This well-founded data forms the basis for the mathematical model development, which is also due to the fact that only few information concerning major structure and thermal properties of the LHP system are available and supplementary effective parameters need to be introduced instead. It has been shown that this procedure leads to a satisfying accordance of the experimental data and the numerical results in a first step. Temperature ranges as well as the trends can be reproduced well by the mathematical model. Nevertheless further experimental investigations have to be carried out in order to enlarge the database and to improve the prediction of the structural and thermal properties and parameters introduced. In addition, the mathematical model will be generalized to be applied to transient conditions as well, in regards to the later application.

\section{Acknowledgements}

The authors would like to thank the German Aerospace Center (DLR) and Tesat-Spacecom GmbH \& Co. KG for their support. 


\section{References}

[1] W. G. Anderson, P. M. Dussinger, S. D. Garner, J. R. Hartenstine and D. B. Saraff, "Loop heat pipe design, manufacturing, and testing - an industrial perspective," Proceedings of the ASME 2009 Heat Transfer Summer Conference, San Francisco, vol. 3, pp. 497-503, 2009.

[2] J. Ku, "Methods of Controlling the Loop Heat Pipe Operating Temperature," SAE Technical Paper 2008-01-1998, 2008.

[3] Y. F. Maydanik, "Loop heat pipes," Applied Thermal Engineering, vol. 25, pp. 635-657, 2005.

[4] JCGM 100:2008, "Evaluation of measurement data - Guide to the expression of uncertainty in measurement," 2008.

[5] K. Cheung, T. Hoang, J. Ku, T. Kaya, "Thermal Performance and Operational Characteristics of Loop Heat Pipe (NRL LHP)," SAE Technical Paper 981813, 1998.

[6] Y. Chen, M. Groll, R. Mertz, Y.F. Maydanik, S.V. Vershinin, "Steady-state and transient performance of a miniature loop heat pipe," International Journal of Thermal Sciences, vol. 45, no.11, pp. 1084-1090, 2006.

[7] Y. B. J. Huang, H. H. Huang, T. L. Liang, "System dynamics model and startup behavior of loop heat pipe," Applied Thermal Engineering, vol. 29, pp. 2999-3000, 2009. 